\title{
College Students’ Transfer from Calculus to Physics
}

\author{
Lili Cui, N. Sanjay Rebello and Andrew G. Bennett ${ }^{\#}$ \\ Physics Department, 116 Cardwell Hall, Kansas State University, Manhattan, KS 66506-2601 \\ \# Mathematics Department, 138 Cardwell Hall, Kansas State University, Manhattan, KS 66506-2602
}

\begin{abstract}
This research investigated students' transfer of learning from calculus courses to an introductory physics course. We used semi-structured think aloud interviews to assess the extent to which students transfer their calculus knowledge when solving problems in a physics course. Results indicate that students needed prompting and scaffolding to connect the calculus knowledge with the physics problem.
\end{abstract}

Keywords: physics education research, transfer of learning, calculus.

PACS: 01.40Fk

\section{INTRODUCTION}

Calculus is a necessary course for calculus-based physics which is sometimes referred to as engineering physics. In most U.S. universities, calculus and physics are taught as two separate subjects in their respective departments. Students are usually required to take particular calculus courses prior to taking physics. This study focused on how students retained and transferred the knowledge from their calculus course when solving problems in their physics course, because how students transfer the knowledge learned in their calculus course can be critical to their learning in physics. To assess students' retention and transfer from calculus to physics, we address the following research questions:

- To what extent do students retain and transfer their calculus knowledge when solving problems in introductory physics?

- What difficulties pertaining to the transfer of calculus knowledge do students have while solving physics problems?

- What strategies may help students overcome the difficulties identified above?

\section{RELEVANT LITERATURE}

Transfer is often defined as the ability to apply what has been learned in one context to a new context (e.g. [1] ). To assess transfer, researchers have often used one-shot assessments such as performance on tests and examinations. Contemporary perspectives describe transfer as a dynamic construction of associations between the two contexts mediated by several factors. [2] For this study, we used think-aloud interviews to assess transfer and the factors that control it.

When assessing transfer from math to physics, Bassok [3] found transfer asymmetry between algebra and physics. Most students who learned algebra only could apply the algebra knowledge to the isomorphic physics problem based upon the targeted algebra concept; however, very few of the students who learned physics alone could apply their physics knowledge to the isomorphic algebra problem. Ozimek [4] examined the retention and transfer from trigonometry to physics. When he assessed transfer using pre-post test gains, Ozimek found no evidence of transfer. However, by using score correlations, based on Lobato's 'Actor-Oriented' transfer [5] he found that students do transfer knowledge from trigonometry to physics.

Integrated curricula have been developed and were found useful in teaching calculus and physics. (e.g. [6]) Yeatts and Hundhausen [7] used their own experiences in talking about the difficulties - "notation and symbolism," "the distraction factor" and "compartmentalization of knowledge" - that students have when transferring their knowledge between calculus and physics and provide some recommendations. However, unlike the integrated curriculum developed by Dunn, [6] calculus and physics are taught as separate subjects in most universities. 


\section{METHODOLOGY}

From contemporary perspectives (e.g. [5]), transfer is considered a dynamic process. So, we conducted semi-structured, one-on-one think aloud interviews to assess how students transfer their calculus knowledge in a physics context.

Engineering Physics (EP) II students who enrolled in Fall 2004 and Spring 2005 at Kansas State University (KSU) participated in this study. We chose EPII because it requires a significant application of differential and integral calculus. EP is a twosemester, calculus-based physics course. Students typically enroll in at least one calculus course before they take any EP course. Approximately $80 \%$ of students enrolled in EP are engineering majors.

Each participant was interviewed over two sessions, each lasting about one hour. The interviewee was left alone to solve an assigned problem. Upon completion, we asked them to explain what they had written down and encouraged them to verbalize their thinking process. We also asked them to describe any difficulties they had when solving the problem. We asked general questions about their calculus background and application of their calculus knowledge in physics at the end of the interview.

We asked interviewees to solve physics problems that were similar to their homework or exam problems and required use of simple integration or differentiation. The four physics problems we used were: (1) E field caused by a half-circle charge distribution, (2) electric potential caused by changing E field, (3) B field caused by a non-uniform current distribution and (4) current induced in a wire loop when the loop is moved through the field produced by a straight current-carrying wire. The study was completed in two phases as described below.

\section{Phase I-Fall 2004}

Eight male volunteers were paid to participate in the interviews. Interviewees were asked to solve four sets of two problems each. Each set consisted of a physics problem and an isomorphic calculus problem that utilized the same calculus concept. The goal was to identify the extent to which students would connect the two problems. The problems also provided a context within which to discuss the overall connections between physics and calculus, as seen from the students' perspective.

\section{Phase II-Spring 2005}

Five male and three female paid volunteers participated in the interviews. Based on the results from Phase I (which are discussed later in this paper) we asked the interviewees to solve a physics problem and explored the origin of any difficulties they may have had. After asking the students to describe how they solved the problem, we presented them with variations of the problem that they had recently solved. These variations were used to explore the situations in which interviewees would use "integration" instead of "summation." Three variations of the physics problems are listed below.

Variation I: As a variation of the "E field caused by a half-circle charge distribution" question, we asked interviewees whether they would use the same method if there were several point charges instead of an arcshaped charge distribution. (Figure 1)
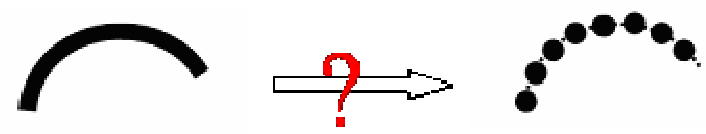

FIGURE 1. Variation I: Uniform charge distribution vs. several point charges

Variation II: As a variation of the "Magnetic field caused by a non-uniform current distribution,” we asked interviewees the difference between the uniform current distribution and a few very thin layers of current. (Figure 2)

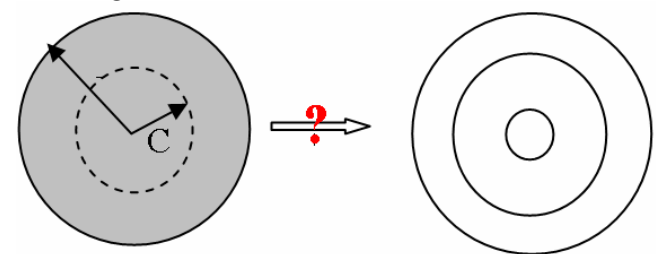

FIGURE 2. Variation II: Uniform current distribution vs. several layers of current

Variation III: As a variation of the "current induced in a wire loop when the loop is moved through the field produced by a straight current-carrying wire" problem, we asked the interviewees the difference for the four cases shown below (Figure 3) with the very small loops.

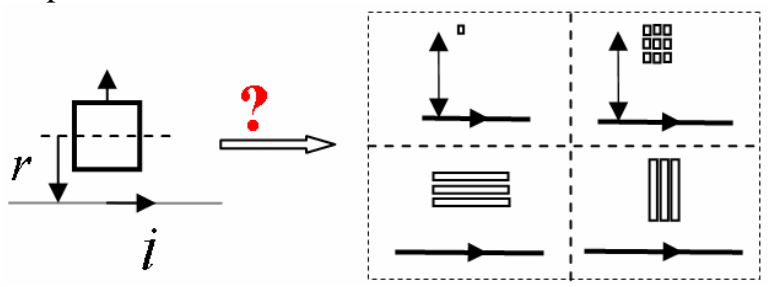

FIGURE 3. Variation III: Single large loop adjacent to wire vs. several smaller loops.

We used phenomenographic approach to analyze all interview data. Phenomenographic analysis [8] yields a variation of students' ideas rather than researchers' conceptions about students' models. The categories for coding of the interactions emerge from 
the analysis of the responses. This strategy is consistent with contemporary views of transfer, such as Lobato's [5]“Actor-Oriented Transfer” model since the researcher does not prejudge what ideas a student might transfer, but rather looks for what, if anything, the student has transferred. The categories from phenomenographic analysis were synthesized using thematic analysis until the dominant themes emerged.

\section{RESULTS AND DISCUSSION}

\section{Phase I-Fall 2004}

The following themes emerged from the analysis of interview data from Phase I.

\section{Self-confidence in calculus knowledge retention}

All interviewees had taken Calculus I and II before taking EP II. Three out of eight interviewees reported positive experiences in their calculus class, three reported negative experiences and others reported neutral experiences. However, all of the interviewees were satisfied and confident of their calculus knowledge. They were successfully able to solve the calculus problems.

\section{Realization that calculus is required in physics}

All of the students realized that physics and mathematics were inextricably linked. As one student commented: "Physics talks about why to solve it, math talks about how to solve it." They also realized that they needed calculus knowledge to solve the physics problems. Seven out of eight interviewees thought their calculus knowledge was sufficient for them to use in physics class. But, students were evenly split when asked whether it would be possible to set up the physics problems without calculus. Furthermore, when asked to compare the calculus and physics problems, only the students who successfully solved the physics problem could see the similarities in the problems. Solving the calculus problem did not help interviewees solve the physics problem.

Lack of confidence in setting up physics problems All of the students had previously seen physics problems similar to the interview question. However, none of them were confident of their physics solution strategy. Students were particularly unclear about the criteria that determined whether calculus should be applied in a given problem.

\section{Phase II-Spring 2005}

In Phase I we had identified that students' difficulties in problem solving in EPII were mainly concerned with setting up the calculus-based physics problem rather than with the calculus per se. In Phase II, we explored these difficulties using the three problem variations described earlier, which helped us explore situations in which students believed integration was applicable to a physics problem. The following themes emerged from Phase II analysis.

Situations in which students use integration in physics

Seven out of eight interviewees appropriately used integration to solve the physics problems, while one student did not use calculus even after being given several hints. When the students that used calculus were asked about the criteria they applied to decide why calculus was applicable to the problem, four out of seven interviewees said the problems were similar to the examples they had seen in the text; however, they could not explain why they used integration. Three out of seven interviewees had a rough idea as to why they needed to use integration in terms of adding up the infinitesimally small elements: "...you can not add up an infinite number...then I used integral...” Interviewees commented that they had not received any specific formal instruction on this topic. Almost all of the students, even those who did not articulate the situations in which they would use integration, could solve the Variation I problem described earlier. Very few interviewees could correctly solve the Variation II and III problems.

Difficulties when applying integrals in physics

The following themes emerged in students' responses when asked about difficulties in applying integrals:

Determining the variable of integration: All interviewees complained that they had difficulty figuring out the "real" variable that needed to be integrated or differentiated. Some commented that "...all constants (variables), I do not know what I should integrate although I know how to integrate..." Interviewees who figured it out, stated that they "got it from both calculus and physics, just look for whatever is changing..."

Deciding the limits of integration: Most interviewees had difficulties in setting up the limits of integration. About half ascribed the difficulties to the physics class. One remarked that it has "...not really to do with my math class...I know how to integrate it, but it is just figuring out what to integrate." Others felt that the calculus class was to blame "because the physics concept is pretty simple,... it is writing an equation for what I understood that is hard..."

Students' preference to use pre-derived algebraic relationship over calculus representation

Most interviewees tended to use pre-derived formulae rather than using calculus to derive the formulae from 
first principles. This tendency led to several difficulties. For instance, they would directly write:

$$
E=\frac{\mu_{0} i}{2 \pi r}
$$

instead of using

$$
\oint \vec{E} d s=\mu_{0} i_{\text {enclosed }}
$$

and then apply it to derive the algebraic relationship. However, when they used the algebraic formula, they were not aware of the conditions in which the formula was applicable. When we asked interviewees why they preferred using algebra rather than calculus they remarked that typical reasoning is "...more confidently use algebra expression to go straight rather than understand this (calculus)..."

\section{Calculus in physics: Understanding or just 'plug and chug'}

Six out of eight interviewees felt that applying calculus in physics is more or less 'plug and chug.' They said: "I do not need to understand it, just how to do it. And I was doing good this way in calculus..."

Two out of eight interviewees believed they needed to understand calculus or they would be "confused."

\section{Strategies to facilitate transfer between calculus and physics}

When students were asked about how their calculus or physics classes could be reformed to facilitate their learning, the following themes emerged.

More 'word' problems in calculus: Students would prefer more application-oriented problems in calculus to prepare them for future applications.

Learning how to set-up physics problems: Students would prefer more step-by-step scaffolding to help them solve problems in physics.

Focus on understanding: Students would prefer a focus on understanding rather than on memorizing equations "even in calculus, I had to understand why the differentiation of $s^{2}$ equal to 2s...", "why integration and differentiation works."

Course sequencing: Students would prefer to take calculus and physics concurrently because "you will have more opportunities to use and understand it..."

\section{CONCLUSIONS}

Our results indicate that students believe that for the most part their calculus class has provided them with adequate knowledge and skills required for physics. However, solving calculus problems did not help students to solve isomorphic physics problems. Students acknowledged they had difficulties in setting up calculus-based physics problems. These difficulties include deciding the appropriate variable and limits of integration, not being clear about the criteria which determine whether calculus is applicable in a given physics problem; and they tended to use oversimplified algebraic relationships to avoid using calculus because they do not understand the underlying assumptions of the relationships.

Students would prefer more application-oriented problems in their calculus course and better scaffolding to help solve physics problems. Students also seem to believe that a focus on conceptual understanding and concurrent teaching of calculus and physics would facilitate their application of calculus in physics.

Our interview results represented a small portion of students taking these courses, but were helpful in identifying the aforementioned issues. In the next phase we will expand our study to design a quantitative investigation of students' performance on calculus-based physics problems on exams.

\section{ACKNOWLEDGEMENTS}

Work supported in part by NSF grant DUE-0206943.

\section{REFERENCES}

1. Byrnes, J.P., Cognitive Development and Learning in Instructional Contexts. 1996, Boston, MA: Allyn and Bacon.

2. Rebello, N.S., et al., Dynamic Transfer: A Perspective from Physics Education Research, in Transfer of Learning from a Modern Multidisciplinary Perspective, J.P. Mestre, Editor. 2005, Information Age Publishing Inc.: Greenwich, CT.

3. Bassok, M. and K. Holyoak, Interdomain transfer between isomorphic topics in algebra and physics. Journal of Experimental Psychology: Learning, 1989: p. 153-166.

4. Ozimek, D.J. Student Learning, Retention and Transfer from Trigonometry to Physics in 2005 Physics Education Research Conference. 2004. Sacramento, CA: AIP Conference Proceedings.

5. Lobato, J.E., How Design Experiments Can Inform a Rethinking of Transfer and Vice Versa. Educational Researcher, 2003. 32(1): p. 17-20.

6. Dunn, J.W. and J. Barbanel, One model for an integrated math/physics course focusing on electricity and magnetism and related calculus topics. American Journal of Physics, 2000. 68: p. 749.

7. Yeatts, F.R. and J.R. Hundhausen, Calculus and physics: Challenges at the interface. American Journal of Physics, 1992. 60: p. 716.

8. Marton, F., Phenomenography- a research approach to investigating different understanding of reality. Journal of Thought, 1986. 21: p. 29-39. 